\title{
An improved mass-loss description for dust-driven superwinds and tip-AGB evolution models
}

\author{
A. Wachter ${ }^{1,2}$, K.-P. Schröder ${ }^{1}$, J. M. Winters ${ }^{3}$, T. U. Arndt ${ }^{2}$, and E. Sedlmayr ${ }^{2}$ \\ 1 Astronomy Centre, CPES, University of Sussex, Falmer, Brighton BN1 9QJ, UK \\ 2 Technische Universität Berlin, Zentrum für Astronomie und Astrophysik, PN 8-1, Hardenbergstr. 36, \\ 10623 Berlin, Germany \\ 3 Max-Planck-Institut für Radioastronomie, Auf dem Hügel 69, 53121 Bonn, Germany
}

Received 7 November 2001 / Accepted 4 January 2002

\begin{abstract}
We derive an improved description of dust-driven stellar mass-loss for the cool winds of carbon-rich tip-AGB stars. We use pulsating wind models in which the mass loss is driven by radiation pressure on dust grains, for C-rich chemistry. From a larger set of these models, selected for representative dynamical (pulsational velocity amplitude $\Delta v$, period $P$ ) and chemical (the $\epsilon_{\mathrm{C}} / \epsilon_{\mathrm{O}}$ abundance ratio) input parameters, an improved approximative mass-loss formula has been derived which depends only on the stellar parameters (effective temperature $T_{\text {eff }}$, luminosity $L$ and mass $M$ ). Due to the detailed consideration of the chemistry and the physics of the dust nucleation and growth processes, there is a particularly strong dependence of the mass-loss rate $\dot{M}$ (in $M_{\odot} / \mathrm{yr}$ ) on $T_{\text {eff }}: \log \dot{M}=8.86-1.95 \cdot \log M / M_{\odot}-6.81 \cdot \log T / \mathrm{K}+2.47 \cdot \log L / L_{\odot}$. The dependence of the model mass-loss on the pulsational period has explicitly been accounted for in connection with the luminosity dependence, by applying an observed period-luminosity relation for C-rich Miras. We also apply the improved mass-loss description to our evolution models, and we revisit their tip-AGB mass-loss histories and the total masses lost, in comparison to our earlier work with a preliminary mass-loss description. While there is virtually no difference for the models in the lower mass range of consideration $\left(M_{\mathrm{i}}=1.0\right.$ to $\left.\approx 1.3 M_{\odot}\right)$, we now find more realistic, larger superwind mass-loss rates for larger stellar masses: i.e., $\dot{M}$ between $\approx 0.4$ and $1.0 \times 10^{-4} M_{\odot} /$ yr for $M_{\mathrm{i}}$ between 1.85 and $2.65 M_{\odot}$, removing between 0.6 and $1.2 M_{\odot}$, respectively, during the final 30000 yrs on the tip-AGB.
\end{abstract}

Key words. stars: carbon - stars: circumstellar matter - stars: evolution - stars: interiors - stars: late-type stars: mass loss

\section{Introduction}

Strong stellar mass-loss by dust-driven "superwinds" (Renzini 1981) on the tip-AGB has long been identified as a major mechanism to recycle stellar material and to drive the chemical evolution of the galaxy. This applies to stars which do not undergo a supernova explosion $\left(M_{*} \lesssim 8 M_{\odot}\right)$ but, instead, lose several tenths to over one solar mass in a strong "cool" stellar wind during their final $\approx 30000$ years (Peimbert 1981; Kwok 1981) to form a planetary nebula (PN). During this brief phase of its evolution, the star becomes a very cool, strongly dust-enshrouded, long-period variable (LPV) object, and it exhibits mass-loss rates of the order of $10^{-4} M_{\odot} / \mathrm{yr}$, as derived from observations and models of LPVs (e.g., Knapp \& Morris 1985; Winters

Send offprint requests to: A. Wachter, e-mail: a.c.wachter@sussex.ac.uk et al. 2000). Because of the interpretational difficulties encountered with LPVs, there has long been a considerable interest in a theoretical description of tip-AGB mass-loss from both, an atmospheric and an evolutionary point of view.

Early descriptions of dust-driven mass-loss used for evolution models (Bowen 1988; Vassiliadis \& Wood 1993; Blöcker 1995) did not take into account the highly temperature dependent chemistry of the dust-formation process, but considered the main energy input to be related to the pulsations of LPVs, rather then being dependent on the interaction of the radiation field with the freshly formed dust grains in the wind acceleration region. For this reason, the derived dependence of the mass-loss rate on the actual stellar parameters, especially on the effective temperature $T_{\text {eff }}$, was in need of considerable improvement. Nevertheless, the magnitude of the total mass lost on the tip-AGB by those models did already match the 
evolutionary context, i.e., the final-mass/initial-mass relation as derived by Weidemann $(1987,1997)$, as that was just a matter of adopting the right scaling of the mass-loss description (Groenewegen et al. 1995).

A much more sophisticated approach to dust-driven mass-loss has been developed over the past 10 years, in terms of pulsating, hydrodynamic and self-consistent numerical models, which describe in detail the complex physics of a dust-driven wind and the dust-formation process around a cool AGB star (Fleischer et al. 1992; Sedlmayr 1994; Sedlmayr \& Winters 1997), for a given set of stellar parameters and carbon-rich chemistry. Massloss rates are then obtained by averaging the outflow of the time-dependent models.

Since individual wind models are very computer time intensive, a first attempt was made to describe the model mass-loss in terms of the stellar and other input parameters by Arndt et al. (1997), using a multi-dimensional, maximum-likelihood fitting procedure on the set of C-rich model mass-loss rates available at that time. By averaging over the existing range of non-stellar (dynamical and chemical) input parameters, a mass-loss description was obtained which depends on the principle stellar parameters, $T_{\text {eff }}, L$ and $M$, only. That description differs remarkably from other approaches in that it is very sensitive to the effective temperature of the star. In fact, the characteristics of the macroscopic system is strongly influenced by the temperature-sensitivity of the microscopic dustformation process.

In using the mass-loss description derived by Arndt et al. (1997) with tip-AGB evolution models, Schröder et al. (1999) found good agreement with observed properties of "superwinds", and even with some observed short bursts of strong mass-loss, suggesting that the gross picture was about right. Meanwhile, however, there has been progress on several points which has created a need for revising the mass-loss description of Arndt et al. (1997):

- many more wind models have been computed in the meantime;

- minimum requirements for the radiative acceleration have been identified (Schröder et al. 1999; Winters et al. 2000) and under-critical wind models should not be considered;

- the non-stellar parameters have either been brought into a physical context, or have been selected for those values which produce models that match observed objects best (see below).

With consideration of the above points, we here provide an improved mass-loss formula for dust-driven, C-rich winds. We then apply it to tip-AGB evolution models to investigate the significance of the new formula to superwind properties and total mass-loss yields by comparison with our previous work (Schröder et al. 1999; Schröder \& Sedlmayr 2001).
Table 1. Range of the parameters covered by the selected model grid.

\begin{tabular}{|lr|r|}
\hline$M$ & {$\left[M_{\odot}\right]$} & $0.8-1.2$ \\
$T$ & {$[\mathrm{~K}]$} & $2200-3000$ \\
$L$ & {$\left[L_{\odot}\right]$} & $3500-15000$ \\
$P$ & {$[\mathrm{~d}]$} & $104-1000$ \\
\hline
\end{tabular}

\section{Deriving the improved mass-loss formula}

Our description of the mass loss during the tip-AGB is based on an substantially updated set of self-consistent, dynamical wind models for dust-forming, carbon-rich atmospheres. The range of model parameters covered matches the observed range and is given by Table 1 . These models include time-dependent hydrodynamics, (equilibrium) chemistry, (stationary, grey) radiative transfer and carbon dust formation, growth and evaporation processes. A recent description of the wind model code has been given by Winters et al. (2000).

The hydrodynamical wind models used to calculate the mass loss rates treat in detail the circumstellar environment around pulsating long-period variable stars and therefore require the prescription not only of the three fundamental stellar parameters (i.e. stellar mass $M$, effective temperature $T_{\text {eff }}$, luminosity $L$ ) plus the abundance ratio $\epsilon_{\mathrm{C}} / \epsilon_{\mathrm{O}}$ of carbon over oxygen. In addition, two parameters are required for the prescription of the inner boundary condition which simulates a pulsating stellar surface as a means of mechanical energy input. This inner boundary condition is provided by a sinusoidal variation of the innermost gridpoint in the hydro-model, parameterized by a velocity amplitude $\Delta v$ and a pulsation period $P$ ("piston approximation").

From the output of the time-dependent models, a mass-loss rate is obtained by averaging the outflow between $r=30$ and $60 R_{*}$, and over a time span of 20 pulsation periods. So far, the models consider only carbon-rich winds, i.e. $\epsilon_{\mathrm{C}} / \epsilon_{\mathrm{O}}>1$. This ratio determines the amount of carbon available for dust formation, making the reasonable approximation that all oxygen and an equal amount of carbon is locked up in CO molecules.

To obtain a physically relevant representation of the theoretical mass-loss rates given by the large set of computed wind models, we applied the multidimensional maximum-likelihood method (as described by Arndt et al. 1997). The input parameters for each wind model are completely independent of each other. Hence, the data set to be described consists of a maximum of 6 independent $(M$, $T_{\text {eff }}, L, \epsilon_{\mathrm{C}} / \epsilon_{\mathrm{O}}, P$ and $\left.\Delta v\right)$ and one dependent variable $(\dot{M})$ for each model. The mass-loss formula should be preferably simple and is therefore assumed to be linear: $\log \dot{M}_{\text {fit }}=a_{0}+a_{1} \cdot \log x_{1}+\cdots+a_{n} \cdot \log x_{n}$.

In order to also give a mass-loss approximation described only by the three fundamental stellar parameters $M, L$ and $T_{\text {eff }}$, Arndt et al. (1997) simply averaged over the mass-loss rates from models with otherwise differing input parameters. Consequently, their simplified formula, 
which we used for our evolution models in earlier work (Schröder et al. 1999; Schröder \& Sedlmayr 2001), neglects any possible dependence of the mass-loss on $\epsilon_{\mathrm{C}} / \epsilon_{\mathrm{O}}$, $P$ or $\Delta v$.

To explore the dependence on any of these parameters and its significance, we first fitted the full set of models available up to date for all 6 parameters to obtain the power of each parameter in the updated representation. We then used the same multidimensional maximumlikelihood method on appropriately reduced parameter sets to compare the quality of the fits, i.e., taking the correlation coefficient of each mass-loss rate approximation $\log \dot{M}_{\text {fit }}$ and the set of the model mass-loss rates $\log \dot{M}$. If, by dropping a certain parameter, the correlation coefficient decreased significantly, and if a parameter enters the mass-loss formula with a comparatively large power, its significance would need further investigation. In this process, we have given special consideration to the following points:

\section{- Exclusion of under-critical wind models:}

Schröder et al. (1999) found that a minimum luminosity, equivalent to a minimum radiative acceleration (Winters et al. 2000), is required in order to maintain a stable dust-driven wind. Under-critical wind models yield mass-loss deficiencies which differ by at least one order of magnitude from the characteristics of stable mass-loss. This, however, had not yet been considered by Arndt et al. (1997). From all currently available wind models, we now selected only the solutions with sufficient radiative acceleration for a stable wind, yielding the high mass-loss rates typical for the tip of the AGB. Precisely, we considered the ratio $\alpha$ of the radiative acceleration $a^{\mathrm{rad}}$ to the gravitational acceleration $a^{\text {grav }}$ and required that its time averaged value $\langle\alpha\rangle_{\mathrm{t}}$ is bigger than 1 .

- Significance of the carbon-to-oxygen ratio $\epsilon_{\mathrm{C}} / \epsilon_{\mathrm{O}}$ : By including this abundance ratio in the fitting procedure, it turned out that for the models under consideration there is only a slight dependence of $\dot{M} \propto$ $0.32 \cdot \log \left(\epsilon_{\mathrm{C}} / \epsilon_{\mathrm{O}}\right)$. Compared to the other parameters the carbon-to-oxygen ratio thus has the least influence on the mass-loss rate. Also, there is virtually no difference in fit quality between the correlation coefficients with $\epsilon_{\mathrm{C}} / \epsilon_{\mathrm{O}}$ resolved (0.966), or it been dropped (0.965).

For the purposes of this paper, we therefore considered it a reasonable simplification to neglect any such dependence.

\section{- The right choice of the piston amplitude $\Delta v$ :}

The mass-loss characteristics of wind models with otherwise identical parameters show a dependence on the chosen piston amplitude. But only models with physically appropriate values give a realistic description of the mass-loss rate. In fact, an appropriate value of $\Delta v$ can be found, and is constrained sensitively, by
Table 2. Mean $K$ - and $M$-amplitudes and mass-loss rates of a set of C-stars.

\begin{tabular}{lccc}
\hline Name & $\langle\Delta K\rangle^{(1)}$ & $\langle\Delta M\rangle^{(1)}$ & $\dot{M}^{(2)}$ \\
\hline R Scl & 0.29 & 0.35 & $6.6 \mathrm{e}-8$ \\
R Lep & 0.45 & 0.48 & $2.3 \mathrm{e}-7$ \\
R For & 0.84 & 0.76 & $7.8 \mathrm{e}-7$ \\
AFGL 799 & 0.83 & 0.80 & $1.7 \mathrm{e}-6$ \\
AFGL 1062 & 0.91 & 0.71 & $2.4 \mathrm{e}-6$ \\
AFGL 2392 & 1.06 & 1.04 & $1.8 \mathrm{e}-6$ \\
AFGL 1235 & 1.07 & 0.94 & $4.7 \mathrm{e}-6$ \\
IRSV 1519-5115 & 1.16 & 0.84 & $9.2 \mathrm{e}-6$ \\
AFGL 1085 & 1.30 & 1.16 & $5.7 \mathrm{e}-6$ \\
IRC + 10401 & 1.32 & 0.93 & $6.3 \mathrm{e}-6$ \\
AFGL 935 & 1.40 & 1.02 & $2.8 \mathrm{e}-6$ \\
IRC -10502 & 1.41 & 1.11 & $7.0 \mathrm{e}-6$ \\
AFGL 971 & 1.48 & 1.17 & $5.9 \mathrm{e}-6$ \\
AFGL 865 & 1.73 & 1.57 & $1.7 \mathrm{e}-5$ \\
AFGL 3099 & 1.78 & 1.21 & $8.0 \mathrm{e}-6$ \\
AFGL 3068 & 1.90 & 1.14 & $4.8 \mathrm{e}-5$ \\
IRC + 10216 & 2.03 & 1.47 & $1.2 \mathrm{e}-5$ \\
\hline
\end{tabular}

(1) Le Bertre (1992), ${ }^{(2)}$ Le Bertre (1997).

matching the amplitudes of observed infrared lightcurves of pulsating AGB stars, which vary almost linearly with the choice of $\Delta v$.

Table 2 summarizes the amplitudes of the $K$ - and $M$ lightcurves of a sample of C-stars monitored by Le Bertre (1992), as well as the mass loss rates derived from phase-dependent spectral modelling by Le Bertre (1997). It can be seen from this table that those objects of the sample, which have a mass loss rate in excess of $10^{-6} M_{\odot} / \mathrm{yr}$, show $K$ amplitudes ranging between 0.8 and $2 \mathrm{mag}$, and $M$ amplitudes between 0.70 and 1.6 mag. Best matches of these amplitudes are achieved by models with piston amplitudes which all fall in the range of 3 to $6 \mathrm{kms}^{-1}$. For deriving the mass-loss formula, we therefore restricted the set of available models to a choice of the piston amplitude of $\Delta v=5 \mathrm{~km} \mathrm{~s}^{-1}$.

- Dependence on the pulsation period P:

In the initial 6 parameter representation of $\dot{M}$, the $P$ term entered with a power of 0.67 . Also, comparing the fit quality with and without including the pulsational period, there is a significant drop of the correlation coefficient from 0.965 to 0.88 . Consequently, the impact of $P$ on the mass-loss description is too significant to be neglected and must be accounted for.

Since a well observed relation exists for pulsating AGB stars between period and luminosity, it is possible to substitute the period term in the mass-loss approximation by an additional luminosity term. In this context, there are a number of models, which do not fall onto such a period-luminosity relation. However, these give a description of how the circumstellar shell model would respond to different lower boundary conditions, 
by which we can properly account for those specific cases matched by real stars.

In the case of carbon-rich Miras, which are the most appropriate class of tip-AGB objects to compare with our models, the observed relation (Groenewegen \& Whitelock 1996) is $\log P / \mathrm{d}=0.965 \cdot \log L / L_{\odot}-1.05$.

With the consideration of the above points, a set of 58 wind models (given in Table 2) has been selected. For applying the maximum-likelihood method on this final set, we now consider only 4 remaining independent input parameters, i.e., effective temperature $T_{\text {eff }}$, luminosity $L$, actual mass $M$ and pulsation period $P$.

For convenience, these parameters are normalized to physically representative reference values, i.e., $1 M_{\odot}$ for the mass, $2600 \mathrm{~K}$ for $T_{\text {eff }}, 10^{4} L_{\odot}$ for luminosity, and $650 \mathrm{~d}$ for the period.

Based on that final set of models, the following massloss description is obtained:

$$
\begin{aligned}
\log \dot{M}_{\text {fit }}= & -4.52-6.81 \cdot \log \left(T_{\text {eff }} / 2600 \mathrm{~K}\right) \\
& +1.54 \cdot \log \left(L / 10^{4} L_{\odot}\right)-1.95 \cdot \log \left(M / M_{\odot}\right) \\
& +0.959 \cdot \log (P / 650 \mathrm{~d}) .
\end{aligned}
$$

It shows a very good correlation (with a coefficient of $0.965)$ with the set of model mass-loss rates. The typical deviation of $\dot{M}_{\text {fit }}$ from any of the actual rates represented by it, is $\pm 20 \%$. This is demonstrated by Figs. 1a-1c where the logarithmic ratio of the mass loss rates is plotted against $M, T_{\text {eff }}$ and $L$ respectively.

In order to substitute the dependence on $P$, we now apply the above mentioned period-luminosity relation. Transformed to our reference values, it is virtually identical with $\log (P / 650 \mathrm{~d})=0.965 \cdot \log \left(L / 10^{4} L_{\odot}\right)$. We can therefore substitute the period term by an additional power of 0.93 of the luminosity. This finally reduces the approximative mass-loss formula to depend on the three fundamental stellar parameters $M, L$, and $T_{\text {eff }}$, only, but without neglecting significant effects from a dependence of the mass-loss on any other parameters $(P$, in particular):

$$
\begin{aligned}
\log \dot{M}_{\text {fit }}= & -4.52-6.81 \cdot \log \left(T_{\text {eff }} / 2600 \mathrm{~K}\right) \\
& +2.47 \cdot \log \left(L / 10^{4} L_{\odot}\right)-1.95 \cdot \log \left(M / M_{\odot}\right)
\end{aligned}
$$

where $\dot{M}_{\text {fit }}$ is given in units of $M_{\odot} / \mathrm{yr}$.

\section{Application to tip-AGB evolution and revised mass-loss yields}

In order to demonstrate the relevance and significance of the improved mass-loss formula for dust-driven, C-rich winds, we applied it to our evolution models in the exact same way as described by Schröder et al. (1999) and Schröder \& Sedlmayr (2001). In particular, we use the same description of convection and overshooting, the critical luminosity for the onset of dust-driven winds, and of any prior mass-loss.

By comparison, the resulting tip-AGB evolution models and their mass-loss histories are virtually the same in

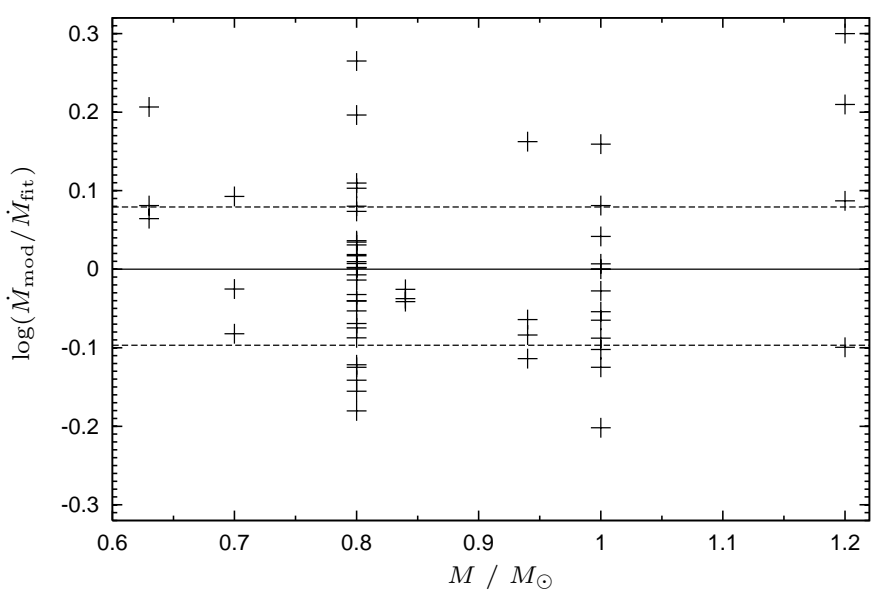

(a) Logarithmic mass loss ratio over initial mass

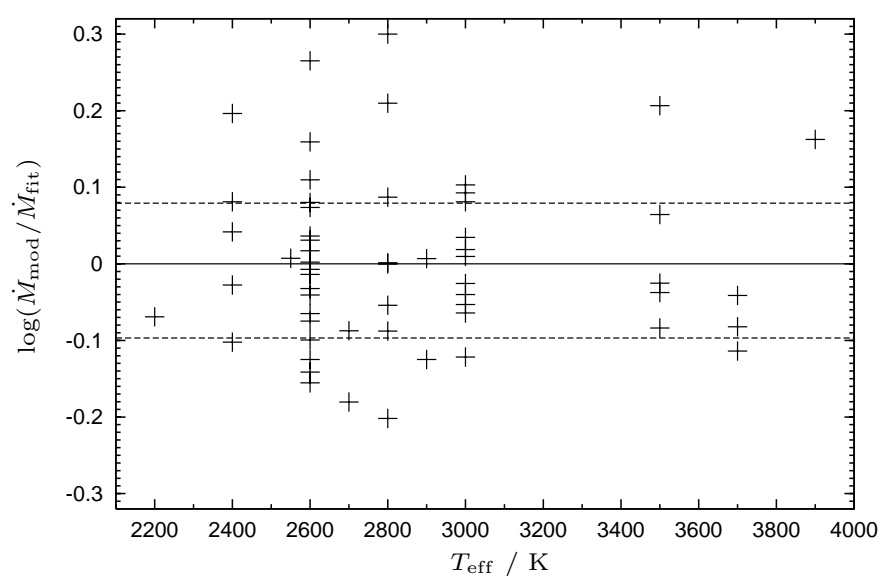

(b) ...over effective temperature

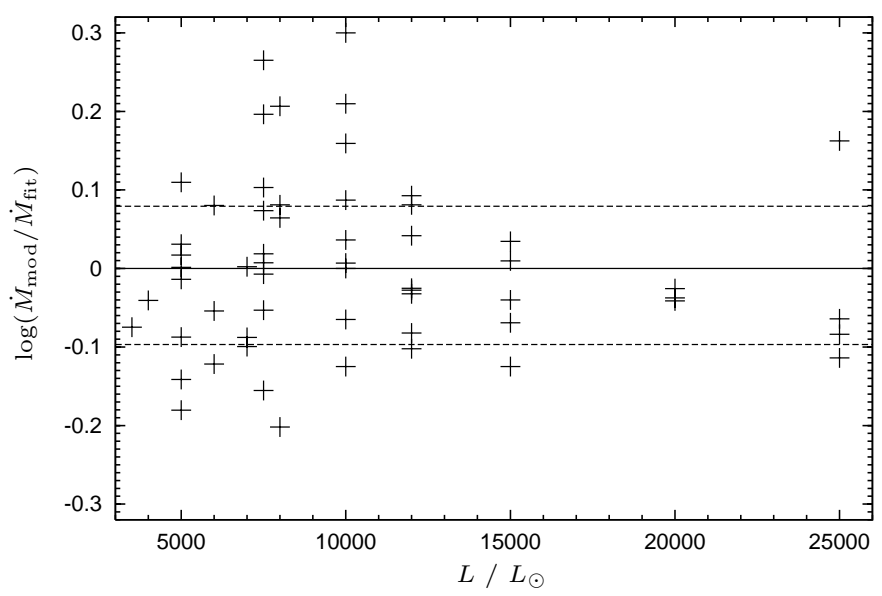

(c) ....and over luminosity

Fig. 1. Deviation of the mass-loss rate given by the fit formula from the model mass-loss rate represented by it. The dashed lines mark a deviation of $20 \%$.

the lower range of initial stellar masses considered here (i.e., $M_{\mathrm{i}}=1.0$ to $\approx 1.3 M_{\odot}$, see Figs. 2,3 ). A proper, 
Table 3. Listing of the 58 wind models, selected by the criteria explained in the text, to realistically represent dust-driven, carbon-rich mass-loss of real tip-AGB objects.

\begin{tabular}{|c|c|c|c|c|c|c|c|c|}
\hline No. & $M_{*} / M_{\odot}$ & $T_{\text {eff }} / \mathrm{K}$ & $L_{\star} / L_{\odot}$ & $P / \mathrm{d}$ & $\langle\dot{M}\rangle$ & $\langle\alpha\rangle_{\mathrm{t}}$ & W-No. & $\epsilon_{\mathrm{C}} / \epsilon_{\mathrm{O}}$ \\
\hline 1 & 0.63 & 3000 & 8000 & 820 & $3.0 \mathrm{e}-5$ & 2.50 & w155 & 1.30 \\
\hline 2 & 0.63 & 3500 & 8000 & 460 & $5.8 \mathrm{e}-6$ & 2.76 & w172 & 1.30 \\
\hline 3 & 0.63 & 3500 & 8000 & 820 & $1.4 \mathrm{e}-5$ & 5.30 & w159 & 1.30 \\
\hline 4 & 0.70 & 3000 & 12000 & 1100 & $6.2 \mathrm{e}-5$ & 6.61 & w156 & 1.30 \\
\hline 5 & 0.70 & 3500 & 12000 & 650 & $1.0 \mathrm{e}-5$ & 4.15 & w174 & 1.30 \\
\hline 6 & 0.70 & 3700 & 12000 & 650 & $6.0 \mathrm{e}-6$ & 1.59 & w176 & 1.30 \\
\hline 7 & 0.80 & 2200 & 15000 & 300 & $1.1 \mathrm{e}-4$ & 5.42 & w38 & 1.30 \\
\hline 8 & 0.80 & 2400 & 7500 & 104 & $1.4 \mathrm{e}-5$ & 3.94 & w125/2 & 1.50 \\
\hline 9 & 0.80 & 2550 & 7500 & 104 & $6.0 \mathrm{e}-6$ & 3.16 & w130/1 & 1.50 \\
\hline 10 & 0.80 & 2600 & 3500 & 400 & $4.9 \mathrm{e}-6$ & 1.30 & w135 & 1.30 \\
\hline 11 & 0.80 & 2600 & 4000 & 400 & $6.5 \mathrm{e}-6$ & 1.38 & w46 & 1.30 \\
\hline 12 & 0.80 & 2600 & 5000 & 300 & $7.4 \mathrm{e}-6$ & 1.34 & w49/14 & 1.30 \\
\hline 13 & 0.80 & 2600 & 5000 & 350 & $6.4 \mathrm{e}-6$ & 1.74 & w110 & 1.30 \\
\hline 14 & 0.80 & 2600 & 5000 & 400 & $1.3 \mathrm{e}-5$ & 1.82 & $\mathrm{w} 44$ & 1.30 \\
\hline 15 & 0.80 & 2600 & 5000 & 500 & $1.3 \mathrm{e}-5$ & 1.88 & w111 & 1.30 \\
\hline 16 & 0.80 & 2600 & 5000 & 600 & $1.6 \mathrm{e}-5$ & 1.96 & w112 & 1.30 \\
\hline 17 & 0.80 & 2600 & 6000 & 400 & $1.6 \mathrm{e}-5$ & 2.28 & w51 & 1.30 \\
\hline 18 & 0.80 & 2600 & 7000 & 450 & $1.9 \mathrm{e}-5$ & 2.61 & w60 & 1.30 \\
\hline 19 & 0.80 & 2600 & 7500 & 300 & $1.0 \mathrm{e}-5$ & 2.38 & w113 & 1.30 \\
\hline 20 & 0.80 & 2600 & 7500 & 450 & $2.5 \mathrm{e}-5$ & 2.61 & $\mathrm{w} 48$ & 1.30 \\
\hline 21 & 0.80 & 2600 & 7500 & 600 & $5.1 \mathrm{e}-5$ & 1.96 & w114 & 1.30 \\
\hline 22 & 0.80 & 2600 & 7500 & 800 & $3.6 \mathrm{e}-5$ & 2.28 & w141 & 1.30 \\
\hline 23 & 0.80 & 2600 & 10000 & 640 & $5.0 \mathrm{e}-5$ & 2.02 & w63 & 1.30 \\
\hline 24 & 0.80 & 2600 & 12000 & 800 & $7.0 \mathrm{e}-5$ & 4.65 & w61 & 1.30 \\
\hline 25 & 0.80 & 2600 & 15000 & 1000 & $9.9 \mathrm{e}-5$ & 3.84 & w62 & 1.30 \\
\hline 26 & 0.80 & 2700 & 5000 & 300 & $3.9 \mathrm{e}-6$ & 1.47 & w167/2 & 1.30 \\
\hline 27 & 0.80 & 2700 & 5000 & 350 & $5.6 \mathrm{e}-6$ & 1.76 & w168/4 & 1.30 \\
\hline 28 & 0.80 & 2800 & 5000 & 400 & $6.1 \mathrm{e}-6$ & 1.26 & w52 & 1.30 \\
\hline 29 & 0.80 & 3000 & 6000 & 400 & $3.8 \mathrm{e}-6$ & 1.85 & $\mathrm{w} 47$ & 1.30 \\
\hline 30 & 0.80 & 3000 & 7500 & 400 & $9.0 \mathrm{e}-6$ & 2.78 & $\mathrm{w} 124 / 2$ & 1.50 \\
\hline 31 & 0.80 & 3000 & 7500 & 450 & $8.3 \mathrm{e}-6$ & 8.49 & w32 & 1.80 \\
\hline 32 & 0.80 & 3000 & 7500 & 650 & $1.0 \mathrm{e}-5$ & 4.86 & w31 & 1.80 \\
\hline 33 & 0.80 & 3000 & 15000 & 300 & $1.7 \mathrm{e}-5$ & 3.70 & w30 & 1.50 \\
\hline 34 & 0.80 & 3000 & 15000 & 650 & $3.0 \mathrm{e}-5$ & 8.90 & w28 & 1.50 \\
\hline 35 & 0.80 & 3000 & 15000 & 800 & $4.1 \mathrm{e}-5$ & 9.32 & w29 & 1.50 \\
\hline 36 & 0.84 & 3000 & 20000 & 1200 & $7.9 \mathrm{e}-5$ & 10.07 & w157 & 1.30 \\
\hline 37 & 0.84 & 3500 & 20000 & 880 & $2.0 \mathrm{e}-5$ & 5.47 & w177 & 1.30 \\
\hline 38 & 0.84 & 3700 & 20000 & 710 & $1.1 \mathrm{e}-5$ & 4.07 & w178 & 1.30 \\
\hline 39 & 0.94 & 3000 & 25000 & 1300 & $8.8 \mathrm{e}-5$ & 11.30 & w158 & 1.30 \\
\hline 40 & 0.94 & 3500 & 25000 & 1000 & $2.3 \mathrm{e}-5$ & 6.77 & w180 & 1.30 \\
\hline 41 & 0.94 & 3700 & 25000 & 810 & $1.2 \mathrm{e}-5$ & 2.85 & w181 & 1.30 \\
\hline 42 & 0.94 & 3900 & 25000 & 1300 & $2.5 \mathrm{e}-5$ & 11.90 & w183 & 1.30 \\
\hline 43 & 1.00 & 2400 & 12000 & 300 & $2.6 \mathrm{e}-5$ & 3.15 & w122/1 & 1.30 \\
\hline 44 & 1.00 & 2400 & 12000 & 500 & $5.9 \mathrm{e}-5$ & 4.04 & w144 & 1.30 \\
\hline 45 & 1.00 & 2400 & 12000 & 600 & $7.7 \mathrm{e}-5$ & 4.09 & w120 & 1.30 \\
\hline 46 & 1.00 & 2400 & 12000 & 800 & $7.9 \mathrm{e}-5$ & 3.71 & w145 & 1.30 \\
\hline 47 & 1.00 & 2600 & 10000 & 640 & $4.3 \mathrm{e}-5$ & 2.64 & w64 & 1.30 \\
\hline 48 & 1.00 & 2600 & 10000 & 650 & $2.6 \mathrm{e}-5$ & 8.78 & w13 & 1.80 \\
\hline 49 & 1.00 & 2800 & 6000 & 400 & $4.6 \mathrm{e}-6$ & 1.41 & w169/5 & 1.35 \\
\hline 50 & 1.00 & 2800 & 7000 & 400 & $5.4 \mathrm{e}-6$ & 1.21 & w78 & 1.30 \\
\hline 51 & 1.00 & 2800 & 8000 & 400 & $5.1 \mathrm{e}-6$ & 1.68 & w97/1 & 1.30 \\
\hline 52 & 1.00 & 2800 & 10000 & 640 & $1.8 \mathrm{e}-5$ & 2.92 & w69 & 1.30 \\
\hline 53 & 1.00 & 2900 & 10000 & 578 & $1.3 \mathrm{e}-5$ & 3.09 & w71 & 1.30 \\
\hline 54 & 1.00 & 2900 & 10000 & 578 & $9.6 \mathrm{e}-6$ & 1.98 & w72 & 1.25 \\
\hline 55 & 1.20 & 2600 & 7000 & 400 & $6.1 \mathrm{e}-6$ & 1.05 & w53 & 1.30 \\
\hline 56 & 1.20 & 2800 & 10000 & 400 & $1.3 \mathrm{e}-5$ & 2.90 & w109 & 1.80 \\
\hline 57 & 1.20 & 2800 & 10000 & 400 & $1.6 \mathrm{e}-5$ & 3.37 & w108/6 & 1.50 \\
\hline 58 & 1.20 & 2800 & 10000 & 400 & $9.8 \mathrm{e}-6$ & 2.06 & w126 & 1.40 \\
\hline
\end{tabular}




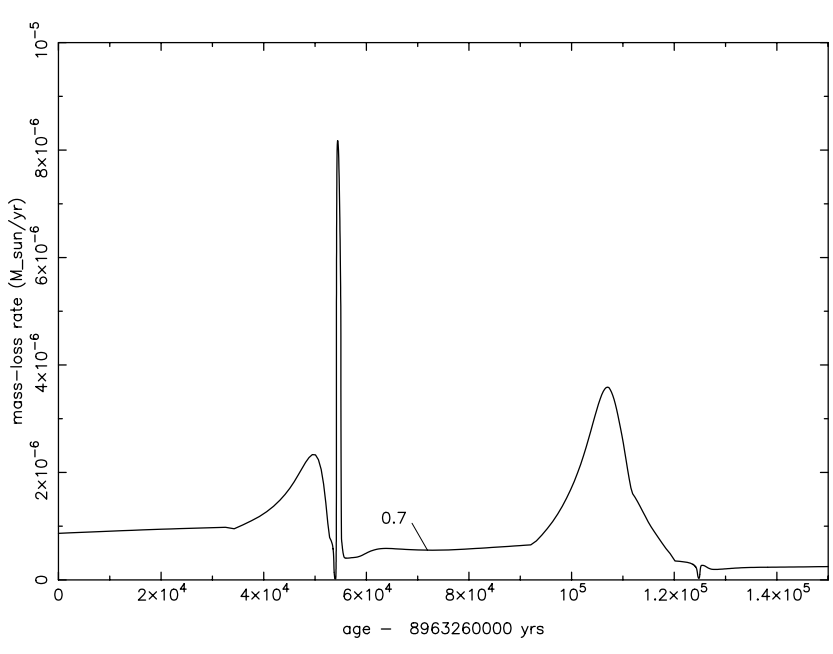

Fig. 2. Tip-AGB and final $10^{5}$ yrs of mass-loss history for a C-rich evolution model with $M_{\mathrm{i}}=1.10 M_{\odot}$ and the improved mass-loss description - note the short burst of mass-loss as already discussed by Schröder et al. (1998, 1999). The label denotes the actual mass (in $M_{\odot}$ ) at that time.

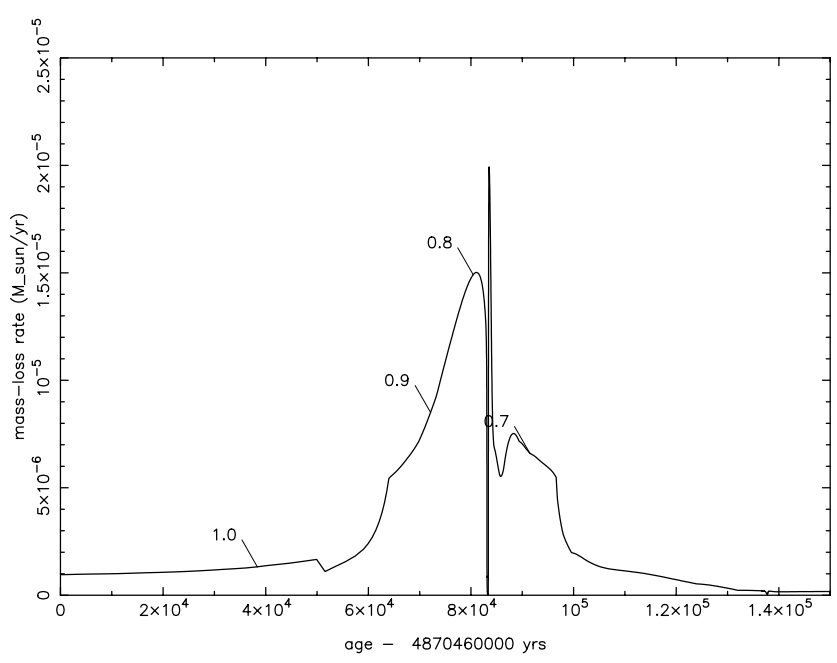

Fig. 3. As in Fig. 2, but for $M_{\mathrm{i}}=1.30 M_{\odot}$, with the improved mass-loss description. This is the minimum initial mass required to obtain a regular superwind.

continuous superwind sets in only for initial masses of $\approx 1.3 M_{\odot}$ and larger (as found before), while short bursts of strong mass-loss are obtained for less massive tip-AGB models. Our new model with $M_{\mathrm{i}}=1.1 M_{\odot}$ produces the same short ( $\$ 800$ yrs) burst of mass-loss (nearly $10^{-5} M_{\odot} / \mathrm{yr}$ ), with the same properties as found by us before (Schröder et al. 1999), and removes obout $0.007 M_{\odot}$ in that event. This is in excellent agreement with the latest observations of stunningly thin, spherical CO shells, especially the one of TT Cyg observed by Olofsson et al. (2000), who derive a total shell mass of $0.007 M_{\odot}$. In addition, their observed outflow velocity $\left(12.6 \mathrm{~km} \mathrm{~s}^{-1}\right)$ of the shell is in very good agreement with the outflow velocity of our respective wind model.

Towards larger initial masses (see Figs. 4, 5), we now obtain higher superwind peak mass-loss rates and larger

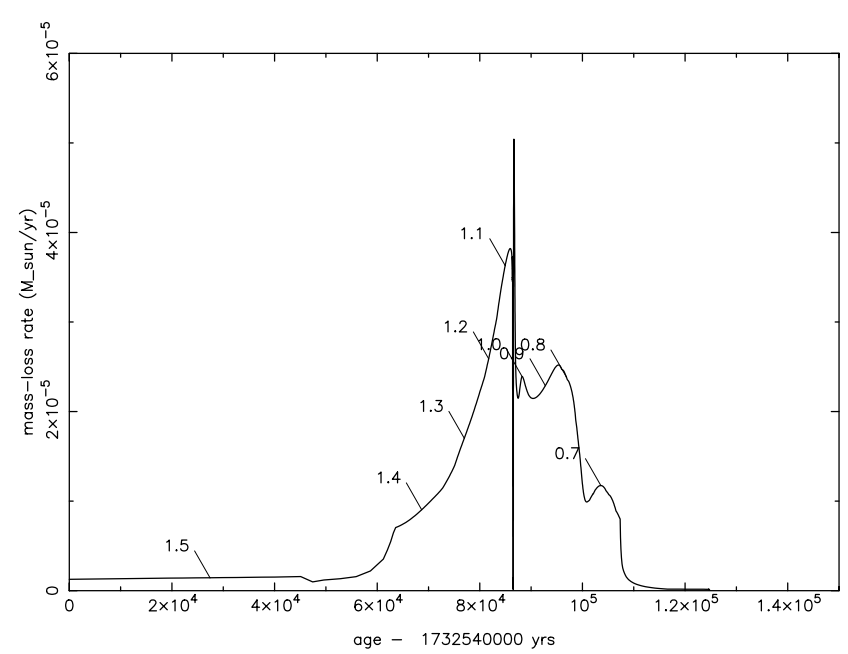

Fig. 4. Tip-AGB and final $10^{5}$ yrs of mass-loss history for a C-rich evolution model with $M_{\mathrm{i}}=1.85 M_{\odot}$ and the improved mass-loss description. Otherwise as Fig. 2.

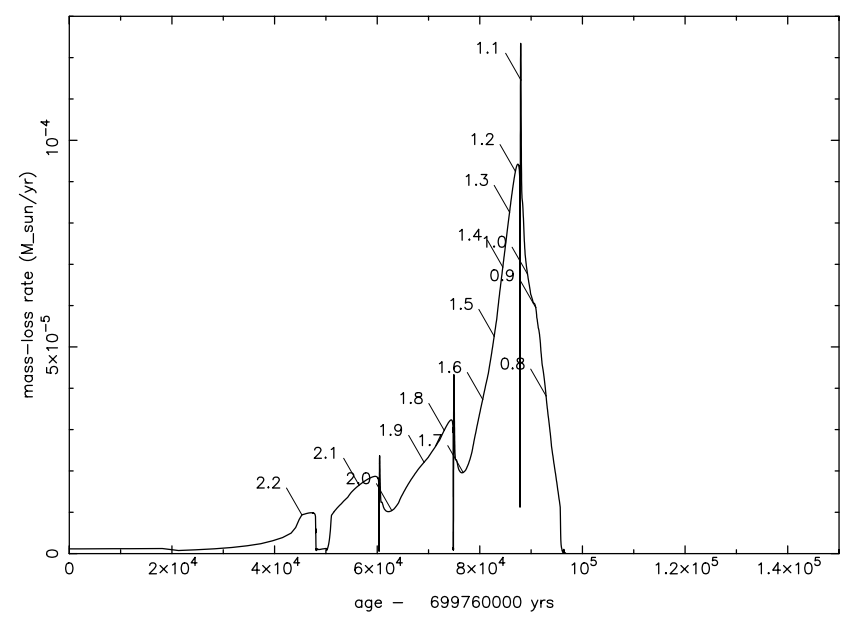

Fig. 5. As in Fig. 4, but for $M_{\mathrm{i}}=2.65 M_{\odot}$. Note the larger peak mass-loss rate for larger initial stellar mass.

fractions of stellar mass lost by the superwind altogether: The $M_{\mathrm{i}}=1.85 M_{\odot}$ model yields $4 \times 10^{-5} M_{\odot} / \mathrm{yr}$ and a total loss of $0.62 M_{\odot}$ during the final 30000 yrs (before: $\left.0.50 M_{\odot}\right)$. The $M_{\mathrm{i}}=2.65 M_{\odot}$ model even develops a superwind mass-loss peak of about $1 \times 10^{-4} M_{\odot} / \mathrm{yr}$, removing $1.22 M_{\odot}$ (before: $0.80 M_{\odot}$ ) in that phase.

The new results agree very well with the characteristics of PNs, and we believe that they now match even better than before. Basically, the new mass-loss description yields larger rates for stars with slightly higher $T_{\text {eff }}$, $M$ and for larger $L$. These stellar parameters are the ones reached by more massive models, which have their tipAGB at higher luminosities and slightly larger $T_{\text {eff }}$. This means, more massive stars produce denser circumstellar shells and, eventually, more massive PNs.

In total, we re-computed 27 C-rich evolution models and their mass-loss histories, for initial stellar masses in the range of 1.0 to $2.8 M_{\odot}$. The basic characteristics of these models are listed in Table 4. This table compares 


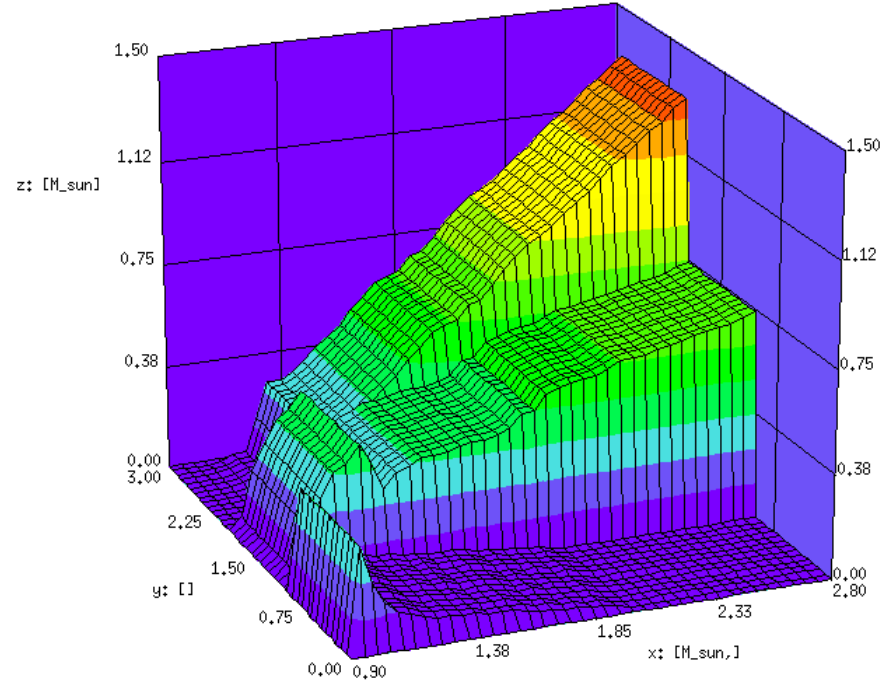

Fig. 6. Mass lost by stars of different initial stellar mass (x-axis) during the RGB (front), the AGB (middle, excl. superwind) and the superwind (back, final $30000 \mathrm{yrs}$ ) phases.

exactly to Table 2 of Schröder \& Sedlmayr (2001). The now stronger mass-loss rates of more massive models lead to significantly larger yields during the superwind phase, which now exceed the AGB mass-losses prior to the superwind, giving more weight to the C-rich superwind yields for their contribution to the galactic mass re-injection rate. The same can be seen from Fig. 6, which compares to Fig. 8 of Schröder \& Sedlmayr (2001), and which shows the total mass lost during each evolution phase relevant to stellar mass-loss, i.e., the Red Gant Branch (RGB), the Asymptotic Giant Branch (AGB, excl. superwind) and the superwind (final $30000 \mathrm{yrs}$ ).

For the same reason, slightly smaller final masses are now obtained, approximately $M_{\mathrm{f}} / M_{\odot}=0.55$. $\left(M_{\mathrm{i}} / M_{\odot}\right)^{0.27}$, which is in even better agreement with the observed initial-mass, final-mass relation of Weidemann (1997) than before: for $M_{\mathrm{i}}=2.5 M_{\odot}$, Weidemann (1997) derived $M_{\mathrm{f}}=0.68 M_{\odot}$, while we obtain $0.70 M_{\odot}$ (instead of $0.72 M_{\odot}$ by Schröder \& Sedlmayr 2001).

Looking at a complete stellar sample of stars, distributed in the range of $M_{\mathrm{i}}=1.0 M_{\odot}$ to $2.5 M_{\odot}$ with a logarithmic IMF representative of the solar neighbourhood stars $\left(\propto M_{\mathrm{i}}^{-1.8}\right.$, see Schröder \& Sedlmayr 2001), we find a re-injection of $57 \%$ of the total stellar mass involved, which is the same fraction as derived before by us (Schröder \& Sedlmayr 2001). Again, however, the difference lies in the mass fraction lost during the C-rich superwind, which we now find to reach nearly $22 \%$ (was $17 \%$ ), while the fraction lost during the prior AGB phases is nearly $29 \%$ (was 33\%). Again, this enhances the importance of the C-rich winds on the tip-AGB relative to the cool but still O-rich winds prior to dust-driven massloss, in terms of their contribution to the galactic mass re-injection.
Table 4. Listing of the revised evolution models, giving the initial stellar mass $M_{\mathrm{i}}$, the masses lost during the RGB, AGB (excl. superwind) and the superwind (final $30000 \mathrm{yrs}$ ) phases, and the final mass $M_{\mathrm{f}}$ of the remnant WD.

\begin{tabular}{cccccc}
\hline \hline$M_{\mathrm{i}}$ & $\int \dot{M}_{\mathrm{RGB}}$ & $\int \dot{M}_{\mathrm{AGB}}$ & $\int \dot{M}_{\mathrm{SW}}$ & $M_{\mathrm{f}}$ & \\
\hline \hline 1.00 & 0.24 & 0.20 & - & 0.55 & \\
1.05 & 0.16 & 0.30 & - & 0.56 & \\
1.10 & 0.12 & 0.38 & 0.01 & 0.56 & 1 \\
1.15 & 0.11 & 0.41 & 0.03 & 0.57 & 1 \\
1.20 & 0.09 & 0.47 & 0.03 & 0.58 & 1 \\
& & & & & \\
1.25 & 0.08 & 0.40 & 0.15 & 0.59 & 1 \\
1.30 & 0.08 & 0.30 & 0.28 & 0.60 & \\
1.35 & 0.07 & 0.35 & 0.28 & 0.60 & \\
1.40 & 0.07 & 0.37 & 0.31 & 0.61 & \\
1.45 & 0.06 & 0.39 & 0.34 & 0.61 & \\
& & & & & \\
1.50 & 0.06 & 0.39 & 0.38 & 0.62 & \\
1.55 & 0.06 & 0.40 & 0.42 & 0.62 & \\
1.60 & 0.05 & 0.41 & 0.46 & 0.63 & 2 \\
1.65 & 0.04 & 0.42 & 0.51 & 0.63 & \\
1.70 & 0.04 & 0.42 & 0.55 & 0.63 & \\
& & & & & \\
1.75 & 0.03 & 0.43 & 0.60 & 0.64 & \\
1.80 & 0.03 & 0.45 & 0.62 & 0.64 & \\
1.85 & 0.02 & 0.50 & 0.63 & 0.64 & \\
1.90 & 0.02 & 0.50 & 0.68 & 0.65 & \\
1.95 & 0.01 & 0.55 & 0.69 & 0.65 & 3 \\
& & & & & \\
2.05 & 0.001 & 0.58 & 0.79 & 0.66 & \\
2.15 & - & 0.59 & 0.87 & 0.67 & \\
2.35 & - & 0.63 & 0.92 & 0.68 & \\
2.50 & - & 0.64 & 1.00 & 0.69 & \\
& -65 & 0.67 & 1.11 & 0.70 & \\
2.80 & - & 0.70 & 1.22 & 0.71 & \\
\hline \hline Sample: & $6.3 \%$ & $28.7 \%$ & $21.7 \%$ & $43.3 \%$ & 4 \\
\hline \hline & & & & & \\
& - & 0.74 & 1.32 & 0.72 & \\
\hline
\end{tabular}

${ }^{1}$ Only brief superwind burst(s).

${ }^{2}$ Onset of core overshooting on MS at $M_{\mathrm{i}} \approx 1.6 M_{\odot}$.

${ }^{3} \mathrm{RGB}$ evolution ends with He flash for $M_{\mathrm{i}} \leq 1.95 M_{\odot}$.

${ }^{4}$ Fractions of mass lost by a stellar sample (1.0 to $2.5 M_{\odot}$, $\left.\mathrm{IMF} \propto M_{*}^{-1.8}\right)$.

\section{Discussion}

Despite significant improvement, there are a few points which will require some more work in the future. In particular, we believe that the most critical of the remaining simplifications concerns the description of the mechanical energy input, $\Delta v$ in particular, for which a fixed value may not be applicable to the whole range of tip-AGB models. Hopefully, a larger set of models, in combination with a better knowledge of stellar parameters of individual pulsating objects, will reveal any kind of relationship between the best choice of $\Delta v$ with any fundamental stellar parameter, i.e., with $L$. Then, any $\Delta v$ term in the mass-loss 
description could be considered in a similar way as we treated the $P$ term.

We would like to emphasize that the here presented mass-loss formula applies only to carbon-rich mass-loss, and for strong dust-driven winds. The chemistry of dustformation in oxygen-rich winds is substantially different and requires its own modelling. Such work is on the way (Jeong et al. 1999) and a specific oxygen-rich mass-loss description is in preparation.

The application of the improved mass-loss approximation to the computation of individual evolution models, as well as to collective mass-loss yields, demonstrates the farreaching implications of any improvement to stellar and galactic astrophysics.

Despite some remaining issues, we may conclude that we have derived a significantly improved and physically meaningful mass-loss description for carbon-rich, dustdriven winds of tip-AGB objects, based on a large set of theoretical wind models, selected to represent observed objects best. The application of the new formula to evolution models yields more massive superwinds and PNs towards the larger stellar masses as derived before with a preliminary mass-loss representation, and the results are in good agreement with observed properties of PNs.

Acknowledgements. This work has been supported by a visitor grant held by A. Liddle, given to JMW for a visit of the Sussex Astronomy Centre, and by a Marie Curie Fellowship of the European Community programme HUMAN POTENTIAL under the contract number HPMT-CT-2000-00096 given to AW to work at the Physics \& Astronomy department of Sussex University. KPS wishes to acknowledge conference travel support, granted to him by the Royal Society.

\section{References}

Arndt, T. U., Fleischer, A. J., \& Sedlmayr, E. 1997, A\&A, 327, 614

Blöcker, T. 1995, A\&A, 297, 727
Bowen, G. H. 1988, ApJ, 329, 299

Fleischer, A. J., Gauger, A., \& Sedlmayr, E. 1992, A\&A, 266, 321

Groenewegen, M. A. T., van den Hoek, L. B., \& de Jong, T. 1995, A\&A, 293, 381

Groenewegen, M. A. T., \& Whitelock, P. A. 1996, MNRAS, 281,1347

Jeong, K. S., Winters, J. M., \& Sedlmayr, E. 1999, in Asymptotic Giant Branch Stars, Proc. IAU Symp. 191, ed. T. Le Bertre, A. Lèbre, \& C. Waelkens (Kluwer Ac. Publ.), 233

Knapp, G. R., \& Morris, M. 1985, ApJ, 292, 640

Kwok, S. 1981, in Physical Processes in Red Giants, ed. Jr. I. Iben, \& A. Renzini (Reidel, Dordrecht), 421

Le Bertre, T. 1992, A\&AS, 94, 377

Le Bertre, T. 1997, A\&A, 324, 1059

Olofsson, H., Bergman, P., Lucas, R., et al. 2000, A\&A, 353, 583

Peimbert, M. 1981, in Physical Processes in Red Giants, ed. Jr. I. Iben, \& A. Renzini (Reidel, Dordrecht), 409

Renzini, A. 1981, in Physical Processes in Red Giants, ed. Jr. I. Iben, \& A. Renzini (Reidel, Dordrecht), 431

Schröder, K.-P., Winters, J. M., Arndt, T. U., \& Sedlmayr, E. 1998, A\&A, 335, L9

Schröder, K.-P., Winters, J. M., \& Sedlmayr, E. 1999, A\&A, 349,898

Schröder, K.-P., \& Sedlmayr, E. 2001, A\&A, 366, 913

Sedlmayr, E. 1994, in Molecules in the Stellar Environment, Proc. IAU Colloq. 146, ed. U. G. Jørgensen (Springer, Berlin), 163

Sedlmayr, E., \& Winters, J. M. 1997, in Stellar Atmospheres: Theory and Observations, Proc. EADN Astrophysics School IX, Lect. Notes Phys. 497, ed. J. P. De Greve, R. Blomme, \& H. Hensberge (Springer, Berlin), 89

Vassiliadis, E., \& Wood, P. R. 1993, ApJ, 413, 641

Weidemann, V. 1987, A\&A, 188, 74

Weidemann, V. 1997, in Advances in Stellar Evolution, ed. R. T. Rood, \& A. Renzini (Cambridge University Press, UK), 169

Winters, J. M., Le Bertre, T., Jeong, K. S., Helling, C., \& Sedlmayr, E. 2000, A\&A, 361, 641 